\title{
Irisin levels increase during experimentally induced acute myocardial infarction
}

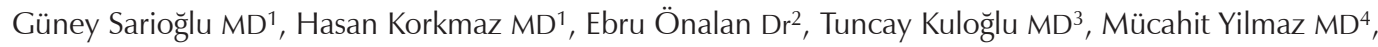 \\ Murat Özgüler MD ${ }^{5}$, Ertuğrul Kurtoğlu MD
}

\begin{abstract}
G Sarioğlu, H Korkmaz, E Önalan, et al. Irisin levels increase during experimentally-induced acute myocardial infarction. Curr Res Cardiol 2016;3(1):9-12.
\end{abstract}

BACKGROUND: Irisin is a hormone involved in energy metabolism. To date, however, there are scant data regarding irisin levels in infarcted tissues. OBJECTIVE: To evaluate changes in irisin levels in the cardiac tissue of rats during experimentally induced myocardial infarction (MI).

METHODS: A total of 21 rats, comprising three groups of seven animals each (MI-1, MI-2 and a control group), were used. Isoproterenol (150 mg/kg once daily for two days ) was used to induce acute MI. The rats in the MI-1 group were evaluated at the end of the first week after the development of MI; rats in MI-2 were evaluated at the end of the second week after the development of MI. Following decapitation, irisin levels in the removed cardiac tissue were examined using polymerase chain reaction and immunohistochemical methods.

T $\mathrm{t}$ is well known that changes in energy metabolism occur under 1 ischemic conditions in acute myocardial infarction (MI) (1). Irisin is a hormone with a peptide structure containing 112 amino acids and is involved in energy metabolism. The basic function of irisin is to transform white adipose tissue into brown adipose tissue, which releases energy in the form of heat. Irisin is fundamentally synthesized from muscular tissue (2).

To date, changes in irisin levels in cardiac muscle during acute MI have not been described. In the present study, we aimed to evaluate irisin levels in the cardiac tissue of rats with experimentally induced MI.

\section{Study population}

\section{METHODS}

A total of 21 adult male albino Wistar rats, eight to 10 weeks of age and weighing between $200 \mathrm{~g}$ and $210 \mathrm{~g}$, were used in the present study. The animals were divided evenly into three groups: MI-1, MI-2 and a control group. All rats were housed in identical cages, which were manufactured specifically for the test, cleaned everyday, and maintained at $21^{\circ} \mathrm{C}$ with $12 \mathrm{~h}$ of light (07:00 to 19:00) and $12 \mathrm{~h}$ of darkness (19:00 to 07:00). Food was dispensed in stainless steel containers and water was provided in glass feeders. The local Animal Experiments Ethics Committee approved the study.

\section{Induction of acute MI}

The rats in group MI-1 received a subcutaneous injection of $150 \mathrm{mg} / \mathrm{kg}$ isoproterenol once per day for two days to induce acute MI. They were decapitated under anesthesia at the end of the first week after MI for further tissue evaluation. The rats in group MI-2 received a subcutaneous injection of $150 \mathrm{mg} / \mathrm{kg}$ isoproterenol once per day for two days to induce acute MI. They were decapitated under anesthesia at the end of the second week after MI for further tissue evaluation.
RESULTS: Following Masson trichrome stain, irisin levels were found to be increased in the cardiac tissue of MI-1 and MI-2 rats compared with the control group. Furthermore, polymerase chain reaction findings demonstrated that messenger RNA levels of irisin increased by a mean ( \pm SD) factor of $23.3 \pm 0.2$ in MI- 1 rats and $15.9 \pm 0.3$ in MI-2 rats compared with the control group ( $\mathrm{P}=0.001$ for both groups). In addition, immunohistochemical methods revealed that the strength and prevalence of irisin immunoreactivity displayed a significant increase in MI-1 rats (+3 strength) and MI-2 rats ( +2 strength) compared with the control group (+1 strength) $(\mathrm{P}=0.01)$.

CONCLUSION: The present study demonstrated an increase in irisin levels in the cardiac tissue of rats with experimentally induced MI. Further studies will determine the significance of this increase in the pathogenesis of MI.

Key Words: Acute myocardial infarction; Irisin; Isoproterenol

The rats in the control group received a subcutaneous sham injection of $1 \mathrm{~mL}$ physiological saline once per day for two days. The animals were decapitated under anesthesia at the same time as the rats in group MI-2 for further tissue evaluation. The diagnosis of MI was made using pathological samples.

\section{Tissue sample preparation}

At the end of the experiment, rats in all groups were decapitated under anesthesia (ketamine $[75 \mathrm{mg} / \mathrm{kg}]$ and xylazine $[10 \mathrm{mg} / \mathrm{kg}]$ ). Following decapitation, cardiac tissue from the rats was quickly removed and fixed in 10\% formaldehyde solution for histological examination. The tissues were stored at $-80^{\circ} \mathrm{C}$ for polymerase chain reaction (PCR) assays.

\begin{abstract}
Histochemical examination
Cardiac tissue removed from all groups were fixed in 10\% formaldehyde fixation solution for $24 \mathrm{~h}$ and irrigated for $24 \mathrm{~h}$ with tap water. Tissues were then administered routine histological follow-up series and embedded in paraffin blocks. Masson trichrome stain was applied to $5 \mu \mathrm{m}$ sections obtained from paraffin blocks. The preparations were then examined using an N-800M (Novel, China) microscope and photographed.
\end{abstract}

\begin{abstract}
Immunohistochemical examination
Irisin expression was observed using immunohistochemistry methods. Sections $5 \mu \mathrm{m}$ to $6 \mu \mathrm{m}$ thick were removed from the parrafin blocks and placed on poly-L-lysine-coated microscope slides. Deparaffinized tissues were passed through a graded alcohol series and boiled for $12 \mathrm{~min}$ in citrate buffer solution with a $\mathrm{pH}$ of 6 in a $750 \mathrm{~W}$ microwave oven for retrieval. After application of Ultra V Block (Lab Vision Corporation, USA) solution for 5 min to prevent ground staining, they were incubated for $60 \mathrm{~min}$ with a primary antibody (rabbit anti-TRPM2
\end{abstract}

${ }^{1}$ Department of Cardiology; ${ }^{2}$ Department of Medical Biology; ${ }^{3}$ Department of Histology and Embryology, Firat University Medicine Faculty; ${ }^{4}$ Department of Cardiology; ${ }^{5}$ Department of Cardiovascular Surgery, Elazig Education and Research Hospital Elazıg, Turkey

Correspondence: Dr Hasan Korkmaz, Department of Cardiology, Frrat University Medicine Faculty, Elazig, Turkey.

Telephone 90-505-351-0740, e-mail hkorkmaz23@hotmail.com 

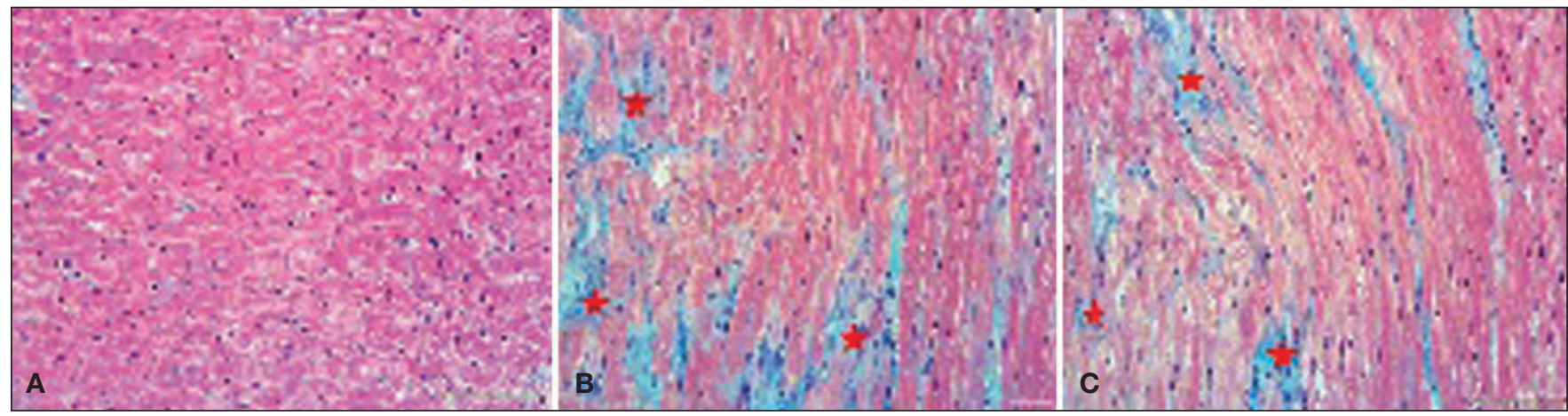

Figure 1) Examination of heart tissue using Masson trichrome stain under light microscopy. A Normal heart histology in control group. B Significant connective tissue increase in heart tissue in myocardial infarction group 1. C Significant connective tissue increase in heart tissue in myocardial infarction group 2
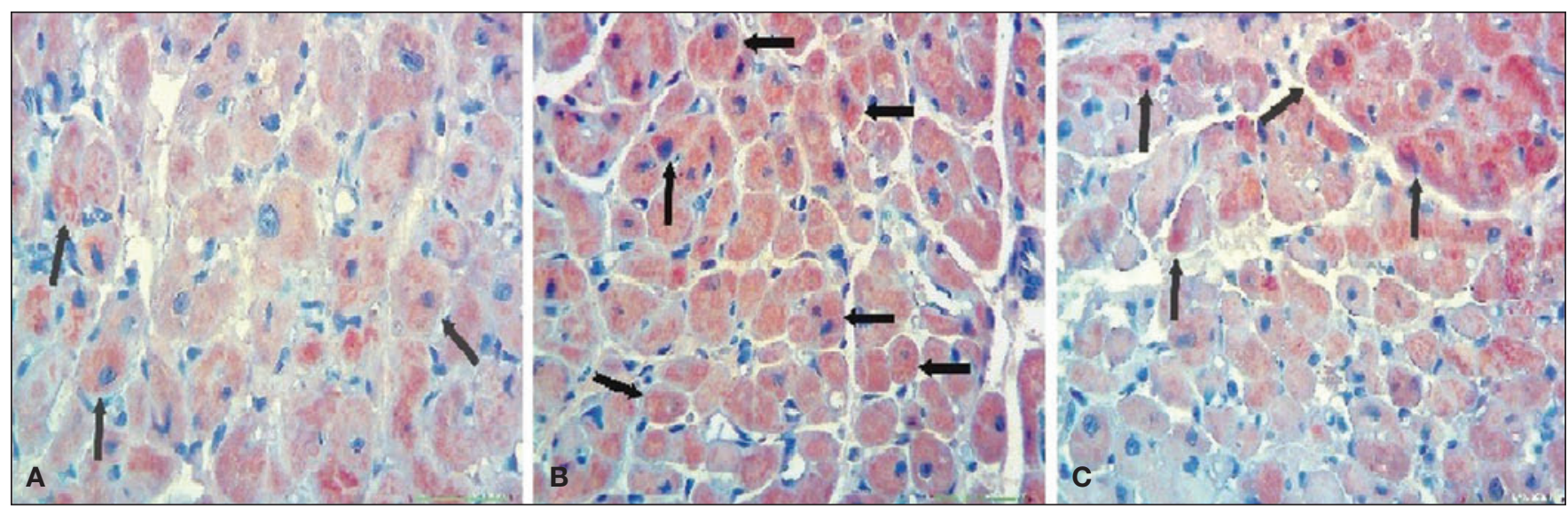

Figure 2) Irisin immunoreactivity. A Irisin immunoreactivity with an average of +1 strength and prevalence in control group heart tissue. $\mathbf{B}$ Irisin immunoreactivity with an average of +3 strength and prevalence in myocardial infarction group 1 heart tissue. $\mathrm{C}$ Irisin immunoreactivity with an average of +2 strength and prevalence in myocardial infarction group 2 heart tissue

antibody, Abcam, United Kingdom). Tissues were then incubated at room temperature in a humid environment with a secondary antibody (biotinylated goat anti-poliyvalent - rabbit immunoglobulin G, Lab Vision Corporation, USA). Subsequently, they were incubated at room temperature in a humid environment with streptavidin alkaline phosphatase (Lab Vision Corporation, USA). They were then placed in distilled water. Fast-Red Substrate System (Lab Vision Corporation, USA) solution was instilled on the tissues and after an image signal was detected using a light microscope, they were taken simultaneously into irrigation with distilled water. After reverse staining using Mayer's hematoxylin, the samples were rinsed with distilled water and occluded using the appropriate occluding solution (Large Volume Vision Mount, Lab Vision Corporation, USA). The resulting slides were examined using a BX 50 (Olympus, Japan) microscope, evaluated and photographed. The evaluation of immunohistochemical staining was based on the prevalence of staining. Prevalence of cytoplasmic immunostaining was scored semiquantitatively by assigning numbers between +1 and +3 : if the staining was low, a score of +1 was assigned; if the staining was moderate, a score of +2 was assigned; and, if the staining was extensive, a score of +3 was assigned.

\section{PCR study}

The Trizol method was used for RNA isolation from cardiac tissue. Cardiac tissue was homogenized with the aid of a homogenizer (Bullet Blender Storm, Next Advance, USA). Isolated RNA samples were stored at $-80^{\circ} \mathrm{C}$ until they were used. RNA measurement (in $\mu \mathrm{g} / \mathrm{mL}$ ) was performed using the Oubit RNA Assay Kit and Oubit 2.0 Fluorometer (Invitrogen, Thermo Fisher Scientific, Australia). An RNA pool was prepared using samples from all groups for each complementary DNA (cDNA) synthesis. Two micrograms of pooled
RNA samples were used for cDNA synthesis. cDNA synthesis was executed using a High-Capacity cDNA Reverse Transcription Kit (Applied Biosystems, USA) in a total volume of $20 \mu \mathrm{L}$. Real-time (RT) PCR assays were performed using rat-specific irisin and glyceraldehyde 3-phosphate dehydrogenase (GAPDH) Tag Man Assays (Applied Biosystems, USA) and Tag Man Master (Applied Biosystems, USA) mix in volume in an ABI Prism 7500 Fast Real Time PCR (Applied Biosystems, USA) thermal cycler. To determine messenger RNA (mRNA) levels of the irisin gene, the GAPDH gene was used as control. Real-time (RT) PCR was implemented in three repetitions. As a result of RT-PCR analyses, the $2^{-\Delta \Delta C T}$ method was used for the calculation of differences in gene expression.

\section{Statistical analysis}

SPSS version 22 (IBM Corporation, USA) was used for statistical analysis. All data were tested for normal distribution using the KolmogorovSmirnov test and were expressed as mean \pm SD. Parametric continuous data were compared between groups using one-way ANOVA, and nonparametric data were analyzed using the Kruskal-Wallis test; $\mathrm{P}<0.05$ was considered to be statistically significant.

\section{RESULTS}

Histochemical findings (Masson trichrome staining)

Samples of cardiac tissue stained with Masson trichrome were examined under a light microscope to observe connective tissue formation; the control group exhibited normal histological appearance. When compared with the control group, a significant increase in irisin levels was observed in the rats in groups MI-1 and MI-2 (Figure 1). 
Immunohistochemical staining for irisin immunoreactivity showed an average of +1 strength and prevalence in the cardiac muscle tissue of the control group (Figure 2A) and an average of +3 strength and prevalence in MI-1 rats (Figure 2B); this difference was statistically significant $(\mathrm{P}=0.01)$. A score of +2 for strength and prevalence for irisin immunoreactivity was reported for rats in group MI-2, which was statistically significant compared with the control group $(\mathrm{P}=0.01)$; however, decreased irisin immunoreacitivity was reported when compared with the MI-1 group (Figure 2C). This decrease was not, however, statistically significant $(\mathrm{P}=0.07)$.

\section{PCR findings}

Irisin mRNA levels are shown in Figure 3. Irisin mRNA levels were $23.3 \pm 0.2$ times higher in group MI-1 compared with the control group $(\mathrm{P}=0.001)$, and $15.93 \pm 0.3$ times higher in group MI- 2 compared with the control group $(\mathrm{P}=0.001)$.

\section{DISCUSSION}

In the present study, irisin immunoreactivity, when compared with the control group, was determined to have increased significantly in both groups of rats with isoproterenol-induced MI (MI-1 and MI-2). Concurrently, in the PCR analysis, it was demonstrated that irisin mRNA levels significantly increased in MI-1 and MI-2 rats.

$\mathrm{MI}$ is the most common cause of death worldwide. Heart cells die due to oxygen deficiency, and necrosis is followed by scar tissue formation (3). Perfusion failure in cardiac tissue results in energy deficiency and loss of contractile activity in oxygen-poor myocardial tissue. Ultrastructural changes are observed in cardiac cells due to prolongation of ischemia and, in internal membranes, cellular integrity is lost and necrosis occurs $(4,5)$.

Isoproterenol, a synthetic symphathomimetic amine (catecholamine), is a molecule structurally similar to adrenalin. It only stimulates $\beta 1$ and $\beta 2$ receptors, and does not stimulate $\alpha$ receptors (6). Isoproterenol is a synthetic catecholamine widely used to induce experimental MI in rats (7). Isoproterenol causes oxygen deficiency and severe myocardial stress, resulting in secondary MI (8). It has been demonstrated that the pathophysiological changes that occur in rat hearts with isoproterenol-induced MI are similar to the changes that occur in the myocardia of humans after MI (9). It has been known that ATP and phosphocreatine levels are reduced post-MI in humans (10). Consistent with earlier data, MI was successfully induced using isoproterenol in our study based on the findings of Masson trichrome staining, in which the control group exhibited normal histological appearance of myocardial tissue, and tissue from MI-1 and MI-2 rats presented increased connective tissue content.

Discovered in 2012 by Bostrom et al (11), irisin is a hormone involved in energy metabolism. It has a peptide structure containing 112 amino acids. Irisin protein is coded by the fibronectin type III domain containing (FNDC 5) gene. Localized at the $1 \mathrm{p} 35.1$ chromosomal region in humans, the gene is localized in the $5 \mathrm{q} 36$ chromosomal region in rats (12). Irisin is usually expressed in heart and skeletal muscles in rodents and humans (13). The total volume of heart muscle especially affects irisin levels (11). Irisin is a new resolver, which increases heat production by breaking the structure of ATP (14). Its basic function is to transform white adipose tissue into brown adipose tissue, to enable energy release in the form of heat. Because of its resolver features, irisin induces heat production via energy molecules such as fats and carbohydrates.

The present study showed that irisin immunoreactivity increased significantly in MI-1 and MI-2 rats. Concurrently, the PCR assay also demonstrated a significant increase in irisin mRNA levels. An empirical study by Kuloğlu et al (15) reported that irisin levels decreased during the first $24 \mathrm{~h}$ of MI. They hypothesized that the decrease in irisin could mitigate ATP loss and provide energy to cells that are in need of energy. The findings of the present study differ from the study by Kuloğlu et al (15). The differences in findings may be related to the length of the study. The study by Kuloğlu et al (15) only examined the

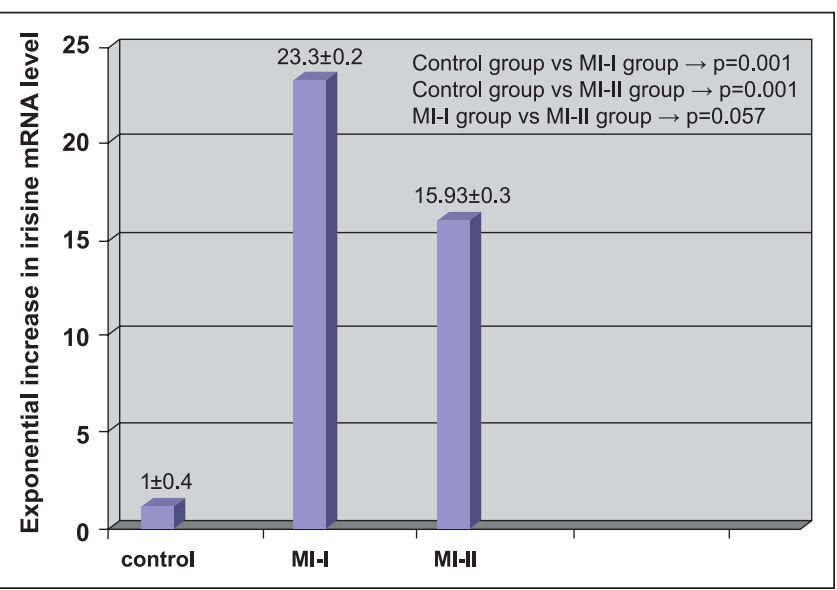

Figure 3) Exponential increase in irisin messenger RNA (mRNA) level. MI-1 Myocardial infarction (MI) group 1; MI-2 MI group 2

first $24 \mathrm{~h}$ after MI occurred and there was no information beyond one week after MI. In addition, a recent study by Aronis et al (16) noted that serum irisin levels had no predictive value in the development of acute coronary syndrome; however, increased irisin levels were related to the development of major adverse cardiac events after coronary intervention. Another study showed that in rats induced with cardiac toxicity using doxorubicin, myocardial and serum irisin levels were increased (17). This study had similarities with our study with respect to the increase observed in irisin level due to myocyte damage.

The increase in irisin levels within the first and second week following MI could also be related to fibroblast activity. It has been known that when irisin levels increase, there is further ATP loss and more heat production. Within the first and second week following MI, fibroblast activity in connective tissue formation increases. It is also been known that ATP consumption is required for connective tissue formation. Therefore, due to the increased need for ATP consumption, a positive feedback mechanism may have increased irisin levels.

In the present study, irisin immunoreactivity and mRNA levels increased after the first week; however, after the second week, they exhibited an insignificant decrease. In fact, this decrease gives rise to the notion that irisin may also be used in MI diagnosis as well as for treatment possibilities. Although the rise in irisin level raises curiosity regarding the possibility of a compensatory situation in which the increase delays myocardial necrosis due to ischemia or to facilitate the repair process, the present study could not clarify this scenario. In addition, this also raises interest about possible treatment options using irisin.

\section{Study limitations}

Because the number of rats used in the present study was limited, a larger sample size would have strengthened the results. In the present study, irisin levels were measured after the first and second weeks following MI. It would have been highly beneficial to observe irisin levels on a daily and weekly basis.

\section{CONCLUSION}

The present study demonstrated that isoproterenol was an appropriate agent for inducing experimental MI; and that MI itself increased irisin levels in cardiac tissue in the first and second week after MI. Future, more detailed studies may demonstrate whether irisin could be used as a biological marker, which could predict tissue damage, aid in prognosis, and determine the role of irisin in MI pathophysiology, diagnosis and treatment.

DISCLOSURES: The authors have no financial relationships or conflicts of interest to declare. 


\section{Sarioğlu et al}

\section{REFERENCES}

1. Rourke RA, Dell'Italia LJ. Diagnosis and management of right ventricular myocardial infarction. Curr Probl Cardiol 2004;29:6-47.

2. Timmons JA, Baar K, Davidsen PK, Atherton PJ. Is irisin a human exercise gene? Nature 2012;488:9-10.

3. Thygesen K, Alpert JS, White HD. Universal definition of myocardial infarction. Eur Heart J 2007;28:2525-38.

4. Kukreja RC, Kontos HA, Hess ML, Ellis EF. PGH synthase and lipoxygenase generate superoxide in the presence of $\mathrm{NADH}$ or NADPH. Circ Res 1986;59: 612-9.

5. van der Vusse GJ, van Bilsen M, Reneman RS. Ischemia and reperfusion induced alterations in membrane phospholipids: An overview. Ann N Y Acad Sci. 1994;723:1-14.

6. Tipnis UR, He GY, Li S, Campbell G, Boor PJ. Attenuation of isoproterenol-mediated myocardial injury in rat by an inhibitor of polyamine synthesis. Cardiovasc Pathol 2000;9:273-80.

7. Benjamin IJ, Jalil JE, Tan LB, Co K, Weber KT, Clarc WA. Isoproterenol-induced myocardial fibrosis in relation to myocyte necrosis. Circ Res 1989;65: 657-70.

8. Yousefi K, Soraya H, Fathiazad F, et al. Cardioprotective effect of methanolic extract of Marrubium vulgare L. on isoproterenolinduced acute myocardial infarction in rats. Indian J Exp Biol 2013;51:653-60.

9. Stelzner TJ, Welsh CH, Berger E, et al. Antiarrhythmic agents diminish thiourea-induced pulmonary vascular protein leak in rats. J Appl Physiol 1987;63:1877-83.

10. Bottomley PA, Wu KC, Gerstenblith G, Schulman SP, Steinberg A, Weiss RG. Reduced myocardial creatine kinase flux in human myocardial infarction: An in vivo phosphorus magnetic resonance spectroscopy study. Circulation 2009;119:1918-24.

11. Boström P, Wu J, Jedrychowski MP, et al. A PGC1-alpha-dependent myokine that drives brown-fat-likedevelopment of white fat and thermogenesis. Nature 2012;481:463-8.

12. Roca-Rivada A, Castelao C, Senin LL, et al. FNDC5/irisin is not only a myokine but also an adipokine. PLoS One 2013;11:e60563

13. Huh JY, Panagiotou G, Mougios V, et al. FNDC5 and irisin in humans: I. Predictors of circulating concentrations in serum and plasma and II. mRNA expression and circulating concen-trations in response to weight loss and exercise. Metabolism 2012; 61:1725-38.

14. Aydin S, Kuloglu T, Aydin S, et al. Cardiac and skeletal muscle serum irisin responses to with or without water exercise in young and old male rats: Cardiac muscle produces more irisin than skeletal muscle. Peptides 2014;52:68-73.

15. Kuloglu T, Aydin S, Eren MN, Yilmaz M, Sahin I, Kalayci M. Irisin: A potentially candidate marker for myocardial infarction. Peptides 2014; 55: 85-91.

16. Aronis KN, Moreno M, Polyzos SA, et al. Circulating irisin levels and coronary heart disease: Association with future acute coronary syndrome and major adverse cardiovascular events. Int J Obes (Lond) 2015;39:156-61.

17. Aydin S, Eren M, Kuloglu T, et al. Alteration of serum and cardiac tissue adropin, copeptin, irisin and TRPM2 expressions in DOX treated male rats. Biotech Histochem 2014;1:1-9. 